\title{
Das urbane Unbewusste
}

\author{
Psychoanalyse und kritische Stadtforschung
}

\author{
Lucas Pohl
}

Anfang der 1990er Jahre haben die anglophonen Geographien damit begonnen, sich mit dem Verhältnis von Psychoanalyse und Stadt auseinanderzusetzen. Ausgehend hiervon kam es Anfang der 2000er Jahre zum Ausruf eines psychoanalytic turn und zur Etablierung von Subdisziplinen, wie den psychoanalytic geographies und der psychoanalytic planning theory, die in den letzten Jahren zu etablierten Bestandteilen der wissenschaftlichen Auseinandersetzung mit Städten im anglophonen Raum geworden sind. Da ein solcher turn hierzulande ausgeblieben ist, stellt sich dieser Beitrag die Frage nach dem Potential einer psychoanalytischen Stadtforschung im deutschsprachigen Raum. Hierzu verfolgt der Autor die These, dass die Stadt bereits in ihrer Entstehung durch das Unbewusste heimgesucht wird. Das urbane Unbewusste kennzeichnet eine Art konstitutiven Störfaktor, der sich in die Topologie der Stadt einschreibt und die Stadt als Objekt (der Stadtforschung) in letzter Instanz unmöglich macht. Ausgehend von dieser Unmöglichkeit, geht der Beitrag den Fantasien rund um die sozialen, politischen und materiellen Verhältnisse einer Stadt nach. Fantasien spielen aus Sicht der psychoanalytischen Stadtforschung eine zentrale Rolle, um der Stadt eine illusorische Konsistenz zu verleihen und das urbane Unbewusste auf Distanz zu halten. Sie ermöglichen es, sich die Stadt vorzustellen, sie zu fühlen und über sie zu sprechen. Der Beitrag endet schließlich mit ein paar Worten zu den Herausforderungen einer künftigen Erschließung der Psychoanalyse für kritische Stadtforschung.

Ersteinreichung: 11. März 2019; Veröffentlichung online: 29. November 2019

An English abstract can be found at the end of the document.

\section{Stadt und Psychoanalyse}

Während einer Konferenz zum Strukturalismus im Jahr 1966 hält der französische Psychoanalytiker Jacques Lacan einen Vortrag in Baltimore, in dem er die Leitlinien seiner Lehre darlegt. Dabei kommt er zu dem Schluss, dass es kein besseres Bild gebe, um „das Unbewusste sinngemäß einzufangen“, als „Baltimore am frühen Morgen“ (Lacan 2015a [1979]: 18). Das Unbewusste lokalisiere sich in den Strukturen der Großstadt. Ein einfacher Blick auf ihre Lichter und den morgendlichen Verkehr lasse die Grenzen zwischen Psyche und Stadt porös werden.

Ich möchte diese Anekdote zum Anlass nehmen, um nach dem Verhältnis von Psychoanalyse und Stadt zu fragen. Eine solche Verknüpfung erscheint aus Sicht der deutschsprachigen Stadtforschung keineswegs selbstverständlich, vielleicht sogar verwunderlich, wenn nicht gar fraglich oder unmöglich.[1] Im Hinblick auf die anglophonen Traditionen der Stadtforschung 
relativiert sich die zunächst abwegig klingende Vorstellung einer psychoanalytischen Stadtforschung. Bereits seit Anfang der 1990er Jahre haben anglophone Geograph_innen damit begonnen, sich mit der Psychoanalyse auseinanderzusetzen (Pile 1991, 1993) und einen „psychoanalytic turn“ (Philo/Parr 2003) ausgerufen, was letztlich zur Etablierung der Subdisziplin psychoanalytic geographies geführt hat, die in Form von Themenheften (Social \& Cultural Geography 2003; The Professional Geographer 2010), Sammelbänden (Kapoor 2018; Kingsbury/Pile 2014a) und Einträgen in Handbüchern (Blum/Nast 2000; Kingsbury 2004) und Enzyklopädien (Bondi 2009; Kingsbury 2009; Pohl 2019a; Proudfoot 2009) zu einem etablierten Bestandteil des geographischen Kanons geworden ist.

Die Stadt hat dabei von Beginn an eine Schlüsselfunktion eingenommen, Geographie und Psychoanalyse miteinander zu vermitteln. Ausgehend von den frühen Arbeiten des britischen Geographen Steve Pile, allen voran seinem einschlägigen Buch The Body and the City (1996), haben in den letzten Jahren eine Reihe von Arbeiten zur Etablierung einer psychoanalytischen Stadtforschung beigetragen (Bullock 2014, 2018; Nichols 2008; Pile 2000, 2005a, 2005b, 2011; Pohl 2017, 2018a, 2018b, 2019b, 2019c; Proudfoot 2011, 2017; Secor 2013). Parallel zu diesen Bestrebungen kam es im Bereich der anglophonen Stadtplanung, speziell in der Planungstheorie, zu einem „psychotheoretical turn“ (Hillier 2003: 44). Damit hat sich hier ebenfalls ein Forschungszweig entwickelt, der sich städtischen Themenund Problemfeldern aus einer psychoanalytischen Perspektive widmet (Davidson 2010; Gunder 2005, 2008, 2011; Gunder/Hillier 2009; Hillier/ Gunder 2003, 2005; Westin 2014).

Doch wie legitimiert sich eine Verknüpfung von Psychoanalyse und Stadtforschung? Henri Lefebvre zufolge kann man der Psychoanalyse einzig dann einen Platz in der Stadtforschung einräumen, wenn man den Beweis liefert, dass die Stadt über ein Unbewusstes verfügt:

„Wenn sich beispielsweise herausstellen sollte, dass jede Gesellschaft, und insbesondere die Stadt, ein unterirdisches und verdrängtes Leben, und somit ein ,Unbewusstes', hätte, würde das abfallende Interesse an der Psychoanalyse sicherlich wieder aufflammen." (Lefebvre 2000 [1974]: 46, Übers. d. A.)

Dass dieser Beweis erbracht werden kann und bereits von der anglophonen Stadtforschung erbracht wurde, werde ich in diesem Beitrag näher erläutern. Hierzu möchte ich jedoch voranstellen, dass das Unbewusste der Stadt nicht als ein ,unterirdisches Leben“ verstanden werden kann. Einer verbreiteten Auffassung zufolge geht es der Psychoanalyse darum, das Verborgene im Untergrund der Psyche freizulegen, um zu verstehen, was an dessen Oberfläche vor sich geht. Demnach läge das Unbewusste räumlich gesehen unter dem Bewusstsein, weshalb es gern auch als ,Unterbewusstes' bezeichnet wird - ein Begriff, der bereits von Sigmund Freud verworfen wurde und bis heute in der Psychoanalyse vollkommen ungebräuchlich ist. Das Unbewusste ist nichts, „was in der Seele unterhalb des Bewusstseins liegt“ (Freud 1991 [1944]: 225) und genauso befindet sich das urbane Unbewusste nicht ,unterhalb' der Stadt. Der Grund, weshalb Lacan das Unbewusste im eingangs aufgeführten Zitat bildhaft in den für alle sichtbaren Strukturen der 
Großstadt (und nicht etwa in den Untiefen der Kanalisation) verortet, liegt darin, dass sich das Unbewusste nicht auf einen bestimmten Ort innerhalb der Stadt beschränkt, sondern gewissermaßen das Herz der Stadt tangiert. So gesehen lässt sich das urbane Unbewusste auch nicht im metaphorischen oder übertragenen Sinne verstehen. Wie ich im Laufe dieses Beitrags darlege, kann eine psychoanalytische Stadtforschung nur dann ernsthaft in Erwägung gezogen werden, wenn wir jeden Zweifel daran ausräumen, dass das Unbewusste einen tatsächlichen Anteil an der (sozialen, politischen und materiellen) Produktion des urbanen Raums trägt.

\section{Psychoanalyse und Gesellschaft(skritik)}

Als Freud Ende des 19. Jahrhunderts damit begann, das Unbewusste in den Fokus der öffentlichen Wahrnehmung zu rücken, war mit einem Schlag klar, dass wir nicht mehr ,Herr im eigenen Haus' sind. Das Unbewusste markierte einen radikalen Bruch mit jeder Vorstellung einer vom Menschen unter selbstbestimmten Bedingungen geschaffenen Welt. Es machte Schluss mit jeder Idee vom Individuum als ein vom freien Willen geleitetes Wesen und ersetzte es durch ein Subjekt, dessen Souveränität über die eigenen Motive grundsätzlich in Frage steht. Kein Wunder, dass diese Erkenntnis eine ernsthafte Krise für all jene bedeutete, die glaubten, dass wir uns im Klaren darüber sind, warum wir Dinge in der Weise denken, sagen und fühlen, wie wir es tun. Für sie bedeutete die Psychoanalyse einen vollständigen Zusammenbruch der bestehenden Bedingungen ihrer Zeit, was die Psychoanalyse zu einem Ereignis im philosophischen Sinne des Wortes werden ließ.

Stellt man sich die Frage, was von diesem Ereignis noch übrig ist, gelangt man schnell zu der Annahme: nicht viel. Freuds bahnbrechende Erkenntnisse und kontroverse Thesen gelten heute weithin als veraltet und wissenschaftlich fragwürdig. Seltsamerweise erweist sich die Psychoanalyse jedoch als ziemlich robust. Wie jedes echte Ereignis verschwindet sie nicht einfach, nachdem sie totgesagt wurde, sondern setzt sich auf eigenwillige Weise in unserem Denken fest und löst somit weiterhin Effekte aus, auch wenn sie nicht mehr in unsere Zeit zu passen scheint. Worauf ich anspiele, ist weniger die Art und Weise, wie Freud in unserer Alltagssprache fortbesteht, zum Beispiel, wenn wir vom ,Freud'schen Versprecher" sprechen, sondern die Beharrlichkeit, mit der die Geistes- und Sozialwissenschaften seit einem knappen Jahrhundert immer wieder zur Psychoanalyse zurückkehren. Die Rezeptionsgeschichte der Psychoanalyse ist in ihrer Vielfalt und Widersprüchlichkeit nur schwer zusammenzufassen. Ich möchte trotzdem den Versuch unternehmen, zumindest zwei Strömungen zu benennen, die das kritische Potential der Psychoanalyse über Jahrzehnte hinweg verteidigt haben und die ich deshalb im weiteren Verlauf für eine Vermittlung von Psychoanalyse und kritischer Stadtforschung für zentrale Orientierungspunkte erachte.[2]

Der Freudomarxismus und die damit verbundene Kritische Theorie in der Tradition der Frankfurter Schule haben seit den 1920er und vor allem bis in die späten 196oer Jahre hinein entscheidende Impulse für eine Vermittlung von Psychoanalyse und kritischer Sozialforschung, speziell marxistischer Gesellschaftstheorie, gesetzt. Die Werke von Wilhelm Reich, Erich Fromm, Max Horkheimer, Theodor W. Adorno, Herbert Marcuse und Walter Benjamin, 
um nur einige Namen zu nennen, wären ohne Freuds Einfluss genauso undenkbar wie ohne den Einfluss von Marx. Grund dieser Vermittlung von Psychoanalyse und Marxismus war die Annahme, dass Freuds Schriften hilfreiche Mittel an die Hand geben, um ,den antagonistischen Charakter der gesellschaftlichen Realität offenbar“ (Adorno 1962: 137) werden zu lassen. Insbesondere die Arbeiten von Walter Benjamin bieten, wie ich im weiteren Verlauf dieses Beitrags zeigen werde, ein großes Potential für die Vermittlung von Psychoanalyse und kritischer Stadtforschung. Wie kein anderer Vertreter der Frankfurter Schule hat sich Benjamin der Großstadt verschrieben (Brenner 2009), und auch wenn er hierzulande in der Stadtforschung keine allzu große Rolle spielt, wird er doch bisweilen als Gründungsmitglied der Stadttheorie aufgeführt (Parker 2004). Insofern Benjamins Philosophie der Stadt in einem entscheidenden Maße von einer gemeinsamen Lektüre von Marx und Freud geprägt ist, wird er zu einem prädestinierten Weggefährten für Diskussionen rund um das Verhältnis von psychoanalytischer und kritischer Stadtforschung.

Eine zweite Strömung, auf die ich hinweisen möchte, geht auf Jacques Lacan zurück.[3] Lacan lehrte von den 1930er bis 1970er Jahren in Paris und führte Freuds Werke in die Welt der französischen Intellektuellen ein. Auch wenn er sein Werk oft als „Rückkehr zu Freud“ (Lacan 2011 [1955]: 9) bezeichnete, geht Lacans Lehre weit über eine Reproduktion oder Aktualisierung von Freuds Schriften hinaus. Vor allem der Bedeutung von Sprache hat Lacan, dem strukturalistischen Zeitgeist seiner Zeit folgend, eine weit größere Bedeutung verliehen. Während Freud in Teilen an einem biologischen Fundament für sein Verständnis des psychischen Apparats festhielt, gründete sich das Subjekt für Lacan auf der Einschreibung in die sozio-symbolische Ordnung. Darüber hinaus hat er, wie kein anderer Psychoanalytiker vor ihm, auf der Rolle der Psychoanalyse jenseits der Klinik beharrt. Während Freud mit Schriften wie Massenpsychologie und Ich-Analyse (1967 [1940]) oder Das Unbehagen in der Kultur (1991 [1944]) bereits in Aussicht stellte, dass die Psychoanalyse nicht auf ihren klinischen Alltag reduziert werden kann, hat Lacan diesen Appell auf ein neues Level gehoben:

„Die Psychoanalyse, wie Lacan sie sah, ist nicht dazu da, uns dabei zu helfen, mit ,unseren' Problemen (im Verhältnis zur Gesellschaft beispielsweise) klarzukommen [...]. Die Psychoanalyse hat eine wesenhaft gesellschaftliche, objektive und kritische Dimension. Es geht nie nur um die Einzelnen und ihre (mehr oder weniger intimen) Probleme - denn diese sind von Anfang an in das sozio-symbolische Feld, das Lacan, den Anderen' nennt, eingeschrieben [...]. Stattdessen besteht die Psychoanalyse nach Lacan darauf, dass der theoretische Umfang der Psychoanalyse die Entwicklung ihrer eigenen Theorie des Subjekts, des Wissens, der Wahrheit, sozialer Bande und anderer theoretischer Gegenstände in angemessener Weise mit einschließt.“ (Zupančič 2009: 8f.)

Ein steter Austausch mit den Werken von Denkern wie Platon, Descartes und Hegel hat Lacan für die Philosophie zum einflussreichsten Psychoanalytiker überhaupt werden lassen. Sein Wirkungsradius ist massiv und reicht von Louis Althusser, Alain Badiou, Étienne Balibar, Ernesto Laclau, Jacques 
Rancière und Slavoj Žižek bis hin zu Judith Butler, Hélène Cixous, Luce Irigaray und Julia Kristeva, um nur eine kleine Auswahl an Referenzen zu nennen. Dabei hat Lacans Ansatz zu anhaltenden Grabenkämpfen in verschiedensten Bereichen der Geistes- und Sozialwissenschaften geführt. Seine Psychoanalyse erschien vor allem vor dem Hintergrund der cultural, linguistic und postmodern turns als zu universalistisch, kontextlos und ahistorisch, um der Komplexität und Pluralität sozialer Prozesse gerecht zu werden. Es ist deshalb kaum verwunderlich, dass die Werke von Henri Lefebvre, Michel Foucault, Judith Butler, Gilles Deleuze und Félix Guattari, welche für die kritische Stadtforschung der letzten Jahrzehnte entscheidende Impulse gesetzt haben, vehemente Kampfansagen gegen die Psychoanalyse, vor allem der Lacanschen Prägung, enthalten.

Bevor ich mich im Folgenden einer psychoanalytischen Stadtforschung im engeren Sinne zuwende, möchte ich einen kurzen Versuch unternehmen, den Universalismus der Psychoanalyse zu verteidigen. Zweifelsfrei wird der Psychoanalyse gerade vonseiten des Poststrukturalismus mit einem gewissen Unbehagen begegnet. Der Grund dafür liegt darin, dass die Psychoanalyse die Welt nicht auf den Diskurs reduziert. Lacan behauptet, dass etwas „der Symbolisierung absolut widersteht“" (Lacan 2015b [1975]: 89). Dieses ,Ding“ bezeichnet er als ,das Reale‘. Die Annahme, dass sich so etwas wie das Reale dem Diskurs widersetzen könnte, erscheint aus Sicht des Poststrukturalismus unmöglich, da bereits die Annahme eines Diskurs-Außerhalb auf eine diskursive Aussage zurückgehe, wie Butler in einer prominenten Kritik darlegt (1997: 257-303). Vonseiten Lacans ist jedoch immer wieder betont worden, dass das Reale nicht einfach außerhalb der sozio-symbolischen Ordnung existiere, sondern vielmehr als immanenter Widerspruch zu dieser Ordnung verstanden werden müsse. Das Reale offenbare „das Geheimnis des Unbewussten“ (Lacan 2015c [1975]: 141) insofern, als dass es die Grenze einer sozio-symbolischen Ordnung markiere (so wie das Unbewusste die Grenze des Bewusstseins markiert). Lacan spricht davon, dass das Reale das sei, was in einer Welt ,nicht geht', insofern es einen Bereich markiert, der nicht den Gesetzmäßigkeiten symbolischen Ordnung gehorcht. Außerdem fügt Lacan hinzu, dass sich die Psychoanalyse mehr als jede Wissenschaft „ganz speziell mit dem [beschäftige], was nicht geht“" (Lacan 2006: 67). Wie Žižek bemerkt, könne das Reale deshalb auch anders ausgelegt werden: Nicht als das, was Lacan vom Poststrukturalismus trennt, sondern als das, was Lacan zum einzig wahren Poststrukturalisten werden lässt:

„Erst mit Lacan tritt der, postmoderne“ Bruch ein, insofern als er einen bestimmten realen, traumatischen Kern thematisiert, dessen Status zutiefst zweideutig bleibt: Das Reale widersteht der Symbolisierung, ist aber gleichzeitig sein eigenes rückwirkendes Produkt. In diesem Sinne könnten wir sogar sagen, dass die Dekonstruktivisten im Grunde genommen noch Strukturalisten sind und dass der einzige Poststrukturalist Lacan ist, der [...] ,das reale Ding' affirmiert, die zentrale Unmöglichkeit, um die jedes symbolische Netzwerk strukturiert ist.“ (Žižek 1991: 142-143, Übers. d. A.)

Adorno zufolge bestand bereits „die Größe Freuds“ (1962: 137) darin, „Widersprüche unaufgelöst stehen“ (ebd.) zu lassen. Einen solch unauflöslichen 
Widerspruch stellt Lacan über die Kategorie des Realen ins Zentrum der Psychoanalyse. Wenn es einen Universalismus der Psychoanalyse gibt, dann besteht dieser nicht einfach darin, jeder Gesellschaft die gleichen allgemeingültigen Prinzipien aufzuzwängen. Das einzig universelle Element der Psychoanalyse ist die Anerkennung eines strukturellen Störfaktors, von dem jede soziale Ordnung heimgesucht wird. Das ultimative Ziel der Psychoanalyse ist es deshalb auch nicht, das Subjekt mit seinem Leid vertraut zu machen und Antworten auf dessen Fragen zu finden, sondern das Subjekt mit der Einsicht zu konfrontieren, dass die sozio-symbolische Ordnung, in die es einschrieben ist - von Lacan als der ,große Andere 'bezeichnet - selbst von Antinomien durchzogen ist und somit keine Lösung für das Subjekt bereitstellt: „Es ist wahr, dass die Psychoanalyse ganz im Zeichen des Anderen steht, aber nur, um festzustellen, dass der Andere nicht existiert, dass er selbst mangelt“ (Dolar 1998: 91, Übers. d. A.). Die Vorstellung, dass Gesellschaften von irgendeinem substantiellen Kern zusammengehalten werden beziehungsweise einer immanenten Konsistenz, Stabilität oder Ordnung unterliegen, ist der Psychoanalyse zufolge eine Fantasie, die sich auf dem Bestreben gründet, das Reale auf Distanz zu halten (Kingsbury 2017). Wie ich im Folgenden darlegen werde, führt uns eine psychoanalytische Perspektive aus Sicht der Stadtforschung letztlich an den Punkt, eine ähnliche Hypothese für die Stadt aufzustellen. Demnach basiert die Annahme von der Stadt, verstanden als eigenständige sozio-symbolische Kategorie, auf einer Fantasie, die nur so lange aufrechterhalten werden kann, wie das Reale auf Distanz gehalten wird. Die psychoanalytische Stadtforschung steht ganz im Zeichen der Stadt, aber nur, um festzustellen, dass die Stadt nicht existiert.

\section{Die topologische Stadt}

Die Behauptung, die Stadt existiere nicht, ruft die prominente These der „planetarischen Urbanisierung“ (Brenner/Schmid 2011) auf den Plan. Mit Bezug auf Lefebvre (2014 [1970]) haben Neil Brenner und Christian Schmid vor ein paar Jahren damit begonnen, für eine Stadtforschung zu argumentieren, die sich von der Stadt als Objekt der Stadtforschung verabschiedet und stattdessen von einem globalen Prozess der Urbanisierung ausgeht. Die Stadt, verstanden als zeitlich und räumlich abgrenzbare Kategorie, sei nicht mehr in der Lage, unsere weltumspannenden urbanen Realitäten abzubilden, da die globalen Urbanisierungsprozesse, die seit den 1980er Jahren bis heute ihren Lauf nehmen, die ganze Welt und nicht nur die Städte tangieren: „Die Kategorie der ,Stadt‘ ist heute als analytisches sozialwissenschaftliches Instrument obsolet geworden“ (Brenner/Schmid 2011: 12, Übers. d. A.).

Die Psychoanalyse, so meine These, erlaubt indes eine anders geartete Verabschiedung von der Stadt als Kategorie der Stadtforschung. Im Gegensatz zur planetarischen Urbanisierung geht eine psychoanalytische Stadtforschung nicht von einer historischen, sondern von einer strukturellen Unmöglichkeit aus, die Stadt als klar abgrenzbares Objekt der Stadtforschung zu bestimmen. Um dieser These nachzugehen, lohnt sich zunächst ein kurzer Blick in die allgemeine Stellung des Raums in der Psychoanalyse. Bereits Freud stellt heraus, dass sich der Raum, mit dem es die Psychoanalyse zu tun hat, nicht entlang der „scharfen Grenzen“ verstehen lässt, „wie sie künstlich 
in der politischen Geographie [des 19. Jahrhunderts] gezogen worden sind“ (Freud 1961 [1944]: 85). Lacan radikalisiert diesen Einwand, indem er der Psychoanalyse einen topologischen Raumbezug unterlegt. Die Topologie steht im Kontrast zur Topographie für ein Raumdenken, das es erlaubt, scharfe Grenzen durch fluide und relationale Grenzziehungen zu ersetzen (Martin/Secor 2014). Eine Figur, die Lacan hierzu vehement ins Spiel bringt, ist das Möbiusband - ein Band, „das es erlaubt, mit größter Leichtigkeit von der Innenseite zur Außenseite überzuwechseln, ohne jemals den Rand zu überschreiten" (Lacan 2010 [2004]: 256). Anhand dieser Figur illustriert Lacan die problematische Trennung des Innerlichen (franz. intimité) vom Äußerlichen (franz. extérieur). Lacan führt den Neologismus ,Extimität ein, weniger um die Grenzen von Innen und Außen einfach zu verwischen, als um darzulegen, wie weit das Subjekt davon entfernt ist, sich selbst am nächsten zu sein (Kingsbury 2007). Die Extimität beziehungsweise „intime Exteriorität“ (Lacan 2016 [1986]: 171) bestimmt das Verhältnis zwischen dem Subjekt und dem Realen. Topologisch betrachtet befindet sich das Reale nicht einfach außerhalb des sozio-symbolischen Feldes, über das das Subjekt sich die Realität erschließt, sondern auf der anderen Seite des Möbiusbandes. Objekte, Orte und Geschehnisse, die von dem Subjekt losgelöst existieren, können insofern einen störenden Effekt auf das Subjekt haben, weil sie topologisch über das Reale an das Subjekt gebunden sind (Pohl 2019b). Dass das Reale folglich nicht im topographischen Raum, sondern im topologischen Raum verortet ist, ist ein zentraler Leitgedanke der psychoanalytischen Geographien (Blum/Secor 2011).

Eine Anekdote zu Rom aus Freuds Das Unbehagen in der Kultur (1991 [1944]) legt bis heute den Grundstein für diesen Gedanken. Auf den ersten Seiten seines Essays gelangt Freud für einen Moment zu der „phantastischen Annahme“, die Stadt Rom sei keine „menschliche Wohnstätte, sondern ein psychisches Wesen“ (ebd.: 427). Detailliert schildert er, wie all die historischen Bauten und Plätze der Stadt zeitgleich übereinander gelagert wären. So wie die Psyche nur verdrängt, doch nicht vergisst, würde auch in der Stadt nichts verschwinden, was nicht durch die Analyse wieder hervorgebracht werden könne. Jeder Versuch einer vollwertigen Demolierung, Ersetzung, Vernichtung des städtischen Raums wäre unmöglich und könne stets zu einer Rückkehr des Verdrängten führen. Während Freud diese Analogie von Stadt und Psyche schnell wieder verwirft, weil sie „zu Unvorstellbarem, ja zu Absurdem“ (ebd.: 428) führe, konstatieren Paul Kingsbury und Steve Pile, dass der Ausgangspunkt psychoanalytischer Geographien darin bestehe, den Implikationen von Freuds Annahme weiter zu folgen. Sie argumentieren für eine Methode der Verschiebung des Blickwinkels von der Topographie hin zur Topologie, um auf das Wirken unbewusster Prozesse und die damit verbundene Entfaltung neuer (topologischer) Räume hinzuweisen (Kingsbury/ Pile 2014b: xvii).

Pile hat diesen Anspruch in seinem Buch Real Cities (2005a) zum Ausgangspunkt seiner Lektüre der Stadt gemacht. Er argumentiert, dass das, was gemeinhin als ,Realität‘ einer Stadt verhandelt wird, oft entlang, harter Fakten' bezüglich deren Lage, Größe, Bevölkerung bestimmt werde. Dem stellt Pile ein Verständnis städtischer Realität gegenüber, welches die imaginären Figuren, optischen Illusionen, irrationalen Ängste und fantastischen 
Geschichten in den Fokus rückt, die Städte seit ihren historischen Ursprüngen bis heute prägen. Im Anschluss an Benjamin argumentiert Pile dafür, die Stadt als Phantasmagorie zu verstehen, ,in die der Mensch eintritt, um sich zerstreuen zu lassen“ (Benjamin 1991a: 50). Die Stadt wird somit weniger durch ihre topographischen als durch ihre topologischen Beziehungen zusammengehalten, wodurch unterschiedliche raumzeitliche Prozesse, Akteure und Orte trotz ihrer gegenseitigen Inkommensurabilität koexistieren können. Parallel hierzu betont Pile, dass die Psychoanalyse dazu anleite, die Stadt als einen „spektralen“ Raum zu verstehen (Pile 2005b), in dem traumatische Ereignisse in Form dessen, was Freud, Wiederholungszwang' genannt hat, wiederkehren können. Illustriert am Beispiel von 9/11 in New York zeigt er, wie ein Ereignis, welches zu einem bestimmten Zeitpunkt an einem konkreten Ort stattgefunden hat, an anderen Orten und zu unterschiedlichen Zeiten wiederkehrt und als eine Art intime Exteriorität auf die traumatischen Dimensionen dieses Ereignisses hinweist.

In ihrem Aufsatz „Topological City“ widmet sich Anna J. Secor (2013) den Konsequenzen einer topologischen Verschiebung des Blickwinkels für die Stadtforschung über das Medium Film. Sie argumentiert, dass eine psychoanalytisch inspirierte Topologie der Stadt dazu anleite, von einer offenen Dialektik auszugehen, durch die Subjekt und Stadt, wie die zwei Seiten des Möbiusbandes, miteinander verbunden sind. Stärker als andere topologische Perspektiven, etwa nach Giorgio Agamben oder Gilles Deleuze, beharrt die Psychoanalyse Secor zufolge darauf, das Subjekt topologisch in der Stadt zu verorten, um dadurch zu einer Auflösung substantieller Vorstellungen von Stadt beizutragen. Sowie Lacan davon ausgeht, dass der große Andere in letzter Instanz nicht existiert, weil der Andere, wie das Subjekt, nicht auf Konsistenz, sondern auf Mangel beruht, existiert auch die Stadt nicht im Sinne einer stabilen, geordneten Struktur. Die Stadt lasse sich, so Secor, nicht auf einen wie auch immer gearteten positiven Zusammenhang hin definieren, sondern könne nur durch eine gemeinsame Unmöglichkeit zusammengehalten werden: „Vielleicht ist die reale Stadt nichts anderes als die Inkommensurabilität unserer Fantasien, die Kluft zwischen unvereinbaren Ansichten“ (ebd.: 439, Übers. d. A.). So wie die andere Seite des Möbiusbands strukturell unerreichbar bleibt, entzieht sich das Reale der Stadt strukturell einer Einordnung in deren sozio-symbolische Ordnung.

\section{Die Illusion der Eigenlogik}

Ausgehend von meiner vorangegangenen Rekonstruktion der topologischen Stadt möchte ich im Folgenden etwas vertiefender auf das ideologiekritische Potential einer psychoanalytischen Stadtforschung eingehen. Im letzten Abschnitt habe ich davon gesprochen, dass sich die psychoanalytische Stadtforschung von einer affirmativen Definition der Stadt verabschiedet, indem sie die Stadt als Teil des Realen begreift. Die Stadt existiert somit für die psychoanalytische Stadtforschung in letzter Instanz nicht, da sie, homolog zum großen Anderen bei Lacan, stets von Antinomien durchzogen ist. Das bedeutet jedoch keineswegs, dass die Stadt nicht trotzdem eine zentrale Funktion in unserer sozialen Realität einnimmt. Die Stadt ist allgegenwärtig, etwa als politische, kulturelle oder ökonomische Kategorie. Dem widerspricht 
die Psychoanalyse auch nicht. Im Gegenteil gestattet sie es, der alltäglichen Vorstellung von Stadt als einheitlichem Objekt eine zentrale Funktion in der Aufrechterhaltung unserer sozialen Realität beizumessen, was zunächst paradox klingen könnte, wo ich doch zuvor darlegte, dass die psychoanalytische Stadtforschung gerade eine solche Vorstellung von Stadt aus den Angeln zu heben versucht.

Um diesen Gedanken weiter nachzuvollziehen, ist es hilfreich, zwei Ebenen zu unterscheiden, über die eine psychoanalytische Stadtforschung operiert: die soziale Realität auf der einen Seite und das Reale auf der anderen Seite des Möbiusbandes. Diese Differenz erlaubt es, trotz der Inexistenz von Stadt auf der Ebene des Realen auf der Wirkmächtigkeit von ,Stadt‘ auf der Ebene der sozialen Realität zu insistieren. Doch zuvor möchte ich ein Zitat voranstellen, welches erlaubt, eine Differenz von Realität und Realem kenntlich zu machen:

„Es wäre falsch, aus der ,Nichtexistenz des großen Anderen', d. h. aus der Tatsache, dass der große Andere nur eine rückwirkende Illusion ist, die die radikale Kontingenz des Realen verdeckt, zu schließen, dass wir diese ,Illusion' einfach aussetzen und die Dinge so sehen könnten, wie sie wirklich sind. Entscheidend ist, dass diese ,Illusion' unsere (soziale) Realität selbst strukturiert: Ihr Zerfall führt zu einem, Verlust der Realität‘.“ (Žižek 1991: 71, Übers. d. A.)

,Illusion' darf im Sinne der Psychoanalyse weder als bloßes Hirngespinst noch als abgründiges oder gar krankhaftes Phänomen verstanden werden. Vielmehr ist Illusion konstitutiv an der Produktion von Realität beteiligt. Insofern jede Realität aus Sicht der Psychoanalyse auf der Illusion von „Vollkommenheit, Integration und Harmonie“ (Stavrakakis 2009: 160) beruht, legt die Psychoanalyse den Grundstein für eine neue Konzeption von Ideologie nach Marx. Neben Althussers Aufsatz „Ideologie und ideologische Staatsapparate“ (2010 [1970]) hat sich Žižek in seinem wegweisenden Frühwerk The Sublime Object of Ideology (1989) ausführlicher diesem Ideologiebegriff gewidmet. Entgegen der verbreiteten Vorstellung von Ideologie als ,falschem Bewusstsein ' postuliert Žižek, dass Ideologie im Sinne der Psychoanalyse eine notwendige Voraussetzung dafür ist, um so etwas wie Realität überhaupt möglich werden zu lassen: „Die Funktion der Ideologie besteht nicht darin, uns einen Fluchtpunkt vor unserer Realität anzubieten, sondern uns die soziale Realität selbst als Flucht vor einem traumatischen, realen Kern anzubieten" (ebd.: 45, Übers. d. A.). Das allgemeine Ziel einer psychoanalytischen Ideologiekritik sei demnach nicht, die Realität hinter der Ideologie aufzudecken, sondern die inhärenten Brüche und Inkonsistenzen, in einem Wort: das Reale, freizulegen, das unsere sozialen Realitäten heimsucht.

Übertragen auf die Stadtforschung, ermutigt die Psychoanalyse somit dazu, zunächst an den Fantasien und Illusionen anzusetzen, die einer Stadt als sozio-symbolischer Einheit ihre Konsistenz und Ordnung verleihen.[4] Was hinter der Grundidee einer „Eigenlogik der Städte“ (Berking/Löw 2008) steckt, wäre aus Sicht einer psychoanalytischen Stadtforschung nichts Geringeres als die Annahme einer sozialräumlichen Fantasie. Der Grund, weshalb sich Hamburg anders anfühlt als Berlin, liegt demnach nicht so sehr 
an einem "gewachsenen Kanon routinierter und habitualisierter Praktiken“ (Löw 2010: 613) als an einer bestimmten sozialen Fantasie, welche die Existenz der beiden Städte als in sich konsistente und voneinander trennbare Gebilde sichert. Diese Fantasie existiert nicht einfach aus sich heraus, sondern muss zunächst hervorgebracht und in ständiger Feinjustierung aufrechterhalten werden. Das eigentliche Ziel einer psychoanalytischen Perspektive besteht jedoch nicht darin, unterschiedliche Stadtkulturen in ihren lokalspezifischen Differenzen festzuschreiben, sondern die Haltbarkeit dieser Festschreibungen infrage zu stellen. Ausgehend von der Annahme, dass es eine soziale Fantasie braucht, um die Kohärenz, Stabilität und Ordnung von Städten zu gewährleisten, rekurriert eine psychoanalytische Stadtforschung auf jene Momente, in denen diese Fantasien brüchig werden und die städtische Realität zu verschwinden droht (Pohl 2017).

Freud schildert ein solches Moment in seinem Aufsatz „Das Unheimliche“ (1966 [1947]). Freud, der mit seiner Analyse des Unheimlichen eine bis heute wegweisende Definition dieses schillernden Begriffs vorgelegt hat, geht in einer Anekdote darauf ein, wie er in den leeren Gassen einer ihm fremden Stadt in Italien spazieren geht. Plötzlich während seines Spaziergangs stellt er fest, dass er sich unbeabsichtigt immer wieder in derselben Gasse einfindet. Dieses Moment assoziiert Freud mit dem Unheimlichen insofern, als es sich hierbei um ein unbeabsichtigtes „Moment der Wiederholung des Gleichartigen“ (ebd. 249) handelt, welches das Subjekt mit einer Art Orientierungsverlust konfrontiert. Hierbei wird der topographische Raum der Stadt um eine topologische Dimension ergänzt, in der die klare Struktur und Ordnung beziehungsweise die ,Identität‘ eines Ortes zusammenzubrechen droht (Pohl 2018c). Diese Anekdote zum Ausgangspunkt nehmend, würde eine psychoanalytische Version des Eigenlogikansatzes uns dazu ermutigen, Momenten der Orientierungslosigkeit in Städten die volle Aufmerksamkeit zu schenken und die unbeabsichtigte Gleichartigkeit von Städten zum Ausganspunkt zu nehmen, um den fragilen und substanzlosen Kern einer jeden Fantasie von Stadt als „Anderssein“ (Löw 2008: 18) freizulegen. Stadtpolitik und Stadtmarketing können sich noch so viel Mühe geben, um der Stadt eine,Identität' zu verleihen, letztlich, so die Annahme einer psychoanalytischen Stadtforschung, bieten sie damit nicht mehr als eine Flucht vor dem Realen, welches jede Eigenlogik der Städte auf Distanz zu halten versucht.

\section{Gebaute Fantasien}

Im letzten Teil meiner Diskussion möchte ich auf die Potentiale einer psychoanalytischen Stadtforschung hinsichtlich der Auseinandersetzung mit gebauten Umwelten eingehen. Es könnte der Eindruck entstanden sein, dass die ,harten' topographischen Dimensionen der Stadt in der psychoanalytischen Stadtforschung zugunsten einer topologischen Perspektive eher vernachlässigt werden. Dies ist keineswegs der Fall. Im Gegenteil, räumt die Psychoanalyse den materiellen Dingen eine entscheidende Funktion ein, wenn es um die Entstehung und Aufrechterhaltung der sozialen Realität geht. Speziell geht es der Psychoanalyse darum zu verstehen, wie Objekte in die Fantasie des Subjekts eingebunden werden. Lacan spricht deshalb davon, dass eine entscheidende Frage der Psychoanalyse darin bestehe nachzuvollziehen, 
wie „aus einem situierbaren, ermittelbaren, austauschbaren Objekt“ jene „Art privates, unmittelbares und dennoch dominantes Objekt“ wird, welches „unser Korrelat im Phantasma ist“ (Lacan 2010 [2004]: 115). Dadurch eröffnet die Psychoanalyse eine Art dritten Weg im Spannungsfeld zwischen linguistic turn und material turn. Weder stellt sie infrage, dass eine physische Welt jenseits des Subjekts existiert, noch gelangt sie dahin, sich dieser Welt losgelöst vom Subjekt zuzuwenden. Im Gegensatz dazu fragt die Psychoanalyse danach, was Dinge befähigt, einen Einfluss auf unser Handeln, Denken und Fühlen zu nehmen, oder anders ausgedrückt: Die Psychoanalyse interessiert sich dafür, wie aus einem externen Objekt ein extimes Objekt wird (Pohl 2019b). Die psychoanalytische Auseinandersetzung mit Materialität trägt somit eine gewisse Nähe zur Marx'schen Analyse der Warenform in sich, insofern es hier nicht so sehr darum geht, die,Dinge an sich ' in den Blick zu nehmen als deren Verflechtung mit der Gesellschaft.

Die Arbeiten von Benjamin bieten eine gute Möglichkeit, um diesen Gedanken weiter zu verfolgen. Nicht zuletzt Benjamins Passagen-Werk (1991a, 1991b), welches aufgrund seines frühen Todes ein Fragment geblieben ist, regt zu Spekulationen darüber an, wie eine psychoanalytische Perspektive auf die gebauten Umwelten der Stadt aussehen könnte. In den Pariser Arkaden sah Benjamin die Relikte „der Traumhäuser der Moderne“ (Pile 2000: 79, Übers. d. A.), die, nachdem sie im 20. Jahrhundert allmählich ihre Funktion verloren haben, verfallene Zeugnisse davon ablegten, mit welcher „latenten Mythologie“(Benjamin 1991b: 1002) die gebauten Umwelten einer Stadt versehen sind. Auf diese Weise geht Benjamin, analog zu der von mir skizzierten Ausgangshypothese der psychoanalytischen Geographien, mit Freud über Freud hinaus, insofern er darauf insistiert, wie die Spuren der Vergangenheit im Stadtraum nicht einfach verschwinden, sondern äquivalent zur Verdrängung der Psyche als materielle Spuren in Form von Ruinen fortbestehen (Featherstone 2005: 313).

Eine vergleichbare Analyse lässt sich mit Bezug auf die Michigan Central Station in Detroit verfolgen. Die Michigan Central Station ist der ehemalige Hauptbahnhof von Detroit und gilt heute als die berühmteste Ruine der USA. Einerseits zentral für die Entstehung des sogenannten ,ruin porn', spielt der einst höchste Bahnhof der Welt andererseits eine zentrale Rolle als nostalgisches Wahrzeichen von Detroits Stadtgeschichte. Am 7. April 2009 verabschiedete der Stadtrat von Detroit einen Beschluss zum Abriss der Michigan Central Station. Nur wenige Monate nach dem Beschluss gründete sich die Michigan Central Station Preservation Society, eine lokale Bewegung, die zu Demonstrationen, Online-Petitionen und gerichtlichen Anzeigen gegen die Stadt Detroit aufrief, um die Abrisspläne aufzuhalten - erfolgreich. Die Idee des Abrisses wurde wenige Monate später fallen gelassen. In einer Rede im Kontext der aktuellen Restauration des Bahnhofs, welcher nach Detroits ,Comeback' im Juni 2018 von der Ford Motor Company gekauft wurde, begründete Detroits Bürgermeister Mike Duggan die damalige Entscheidung des Stadtrates damit, dass es zu diesem Zeitpunkt „keine Hoffnung gab, dieses Gebäude und die Stadt zurückzubringen“, „der Bahnhof war tot und Detroit war tot" (Protokoll aus eigener Feldforschung, 19.06.2018).

Es ist bemerkenswert, dass trotz der scheinbaren Hoffnungslosigkeit bezüglich einer möglichen Revitalisierung der Ruine so viele Menschen aktiv 
an der Rettung des verfallenen Bahnhofs beteiligt waren. Während in den letzten Jahren Zehntausende von Gebäuden in Detroit abgerissen wurden, konnte diese Ruine nicht ohne Widerstand verschwinden. Mithilfe einer psychoanalytischen Perspektive lässt sich der Grund für diesen Aufschrei darauf zurückführen, dass die Michigan Central Station, wie kein anderer Teil der gebauten Umwelt als Objekt der Fantasie in der sozialen Realität Detroits fungiert (Pohl 2019c). ,City of the American Dream', ,Auto Capital', ,Paris of the West', ,Arsenal of Democracy - all diese Referenzen auf eine Zeit, in der Detroit das Zentrum der westlichen Welt zu sein schien, kumulieren in dieser Ruine. Dadurch verkörpert der Bahnhof die latente Mythologie einer „unsterblichen Stadt“ (Pohl 2018b). Die Ruine gestattet es, unabhängig von der jahrelang vorgebrachten Behauptung, Detroit sei am Ende, an einer Fantasie von Unvergänglichkeit festzuhalten, welche den nostalgischen Zeitgeist in der Stadt untermauert. Diese Fantasie war es, die im Moment des drohenden Abrisses 2009 auf dem Spiel stand, und diese Illusion war es, welche die Bewegung rund um die Rettung des verfallenen Bahnhofs verteidigte. Die Verflechtung von Fantasie und Materialität spielt folglich eine entscheidende Rolle, um zu verstehen, wieso der Verlust dieser Ruine so viel mehr zu wiegen schien als der Abriss etlicher anderer Teile der gebauten Umwelt Detroits. Eine ähnliche These lässt sich mit Bezug auf den Brand der Notre Dame im April 2019 aufstellen. Der Grund, weshalb binnen weniger Stunden nach dem Brand mehr als 900 Millionen Euro private Spendengelder für den sofortigen Wiederaufbau der Kirche angekündigt wurden und weshalb der Präsident Frankreichs noch am Abend des Brandes öffentlich den Wiederaufbau der Kirche bekanntgab, liegt aus psychoanalytischer Sicht nicht so sehr in der Erhaltung des Gebäudes selbst als im Erhalt einer bestimmten sozialen Fantasie (von Vollkommenheit, Ordnung, etc.), für die das Gebäude als Träger beziehungsweise Fetisch fungiert.

Eine Perspektive, die die gegenseitige Verflechtung von materiellen und psychischen Dimensionen des Raums anerkennt (Blum/Secor 2011), ermöglicht es der kritischen Stadtforschung, nachzuvollziehen, wieso bestimmte Teile der gebauten Umwelt einer Stadt mehr Gewicht haben als andere. Der Grund, weshalb eine Kirche wie Notre Dame aus politischer Sicht nicht einfach niederbrennen darf (im Gegensatz zum Regenwald beispielsweise), hängt in großen Teilen mit der sozialen Fantasie zusammen, die diesem Objekt innewohnt. Solche „fantasievollen Materialitäten“ (Wilson/Bayón 2017) sind existentiell an der Aufrechterhaltung sozialer Realitäten beteiligt, was sich, wie Benjamin richtig erkannt hat, meist erst dann offenbart, wenn Dinge ihre Funktion als Träger von Fantasien verloren haben und zu überflüssigem Abfall erstarren. Erst wenn Dinge „keine Macht mehr haben über die kollektive Phantasie, ist es möglich, sie als die trügerischen Traumbilder zu erkennen, die sie immer schon gewesen sind“ (Buck-Morss 2000: 199).

\section{Psychoanalyse der Städte}

„Während einige blasierte Geister behaupten, dass wir bereits über die Psychoanalyse hinweg sind, ist die Wahrheit, dass wir ihre revolutionärsten Erkenntnisse immer noch nicht eingeholt haben." (Copjec 2002: 9, Übers. d. A.) 
Eingangs habe ich ein Zitat von Henri Lefebvre zum Ausgangspunkt genommen, um nach den Möglichkeiten einer psychoanalytischen Stadtforschung zu fragen. Demnach sei eine solche Stadtforschung nur denkbar, wenn sich die Existenz eines urbanen Unbewussten beweisen ließe. Im Anschluss an die vorangegangene Diskussion behaupte ich, dass ein solches Unbewusstes tatsächlich existiert, jedoch nicht in der Vorstellung, wie sie Lefebvre im Kopf hatte. Das urbane Unbewusste ist keine tieferliegende, verborgene Sinnstruktur, die uns Aufschluss über die geheimen Antriebe der Stadtbewohner_innen gibt, sondern der Sand im sozialen Getriebe der Stadt. Das urbane Unbewusste ist ein Überschuss, der von den Fantasien rund um die Stadt (als konsistenter, sinnvoller, harmonischer, geschichtsträchtiger, identitätsstiftender usw. Zusammenhang) nicht abgedeckt wird. Es ist eine Art substanzloser und sinnloser Rest, der übrig bliebe, wenn wir der Stadt all ihre Fantasien nehmen würden. Dieser konstitutive Überschuss ist, so die Ausgangshypothese einer psychoanalytischen Stadtforschung, eine Sollbruchstelle, auf die sich jede soziale Realität einer Stadt gründet. Das urbane Unbewusste ist in diesem Sinne die psychoanalytische Antwort darauf, dass jede Stadt von Widersprüchen und Antagonismen durchzogen ist. So wie die marxistische Stadtforschung davon ausgeht, dass jede Stadt ein Ort sozialer Kämpfe ist, geht die psychoanalytische Stadtforschung davon aus, dass die Stadt ein Ort in sich widersprüchlicher Fantasien ist. Insofern stellt sich aus Sicht einer psychoanalytischen Stadtforschung tatsächlich heraus, dass die Stadt über ein Unbewusstes verfügt. Wie das Möbiusband fußt die Stadt, sobald sie als sozio-symbolische Entität in Erscheinung tritt, auf einer topologischen Rückseite, die nicht (oder nicht hinreichend) von den Fantasien rund um die Stadt abgebildet werden kann. Diese Rückseite ist das, was man mit Lacan als das Reale der Stadt bezeichnen kann, eine Art struktureller Störfaktor, der die Stadt als Objekt (der Stadtforschung) in letzter Instanz unmöglich macht. Das allgemeine Ziel einer psychoanalytischen Stadtforschung besteht demnach darin, die Stadt (oder Teilaspekte der Stadt, wie ihre gebauten Umwelten) auf ihre sozialen Fantasien hin zu befragen, um davon ausgehend nach den inhärenten Widersprüchen und Inkonsistenzen zu suchen, von der diese Fantasien heimgesucht werden.

Abschließen möchte ich diesen Beitrag mit ein paar Worten zu den Herausforderungen einer Erschließung der Psychoanalyse für kritische Stadtforscher_innen: Analog zu der Behauptung, dass die Psychoanalyse „die Hoffnung auf eine neue Wissenschaft" (Lacan 2015d [1973]: 25) in Aussicht stellt, haben die psychoanalytischen Geographien seit ihren Ursprüngen hervorgehoben, dass sich die Konzepte der Psychoanalyse nicht problemlos in das geographische Denken integrieren lassen: „Kein Wunder also, dass es keine anerkannten psychoanalytischen Konzepte gibt, die sich leicht in Geographie umsetzen, überlagern oder neben der Geographie abbilden lassen - unabhängig von der Art der Geographie“(Pile 1996: 81, Übers. d. A.).

Vor diesem Hintergrund stellt die bis dato fehlende Einführung in die psychoanalytische Stadtforschung eine große Hürde für eine stadtforscherische Aneignung der Psychoanalyse dar. Auch wenn die bestehenden Arbeiten bereits zentrale Bausteine für eine psychoanalytische Stadtforschung bereitstellen, fehlt es an einer Lektüre, die das Verhältnis von Stadt und Psychoanalyse theoretisch herleitet und die psychoanalytische Stadtforschung systematisch 
ins Verhältnis zu anderen bereits etablierten Spielformen der kritischen Stadtforschung setzt sowie zentrale Konzepte, Methoden und Themenfelder einer psychoanalytischen Stadtforschung auslotet. The Body and the City (Pile 1996) ist sicherlich der Text, der diesem Versuch bisher am nächsten kommt. Jedoch ist das Buch bereits über 20 Jahre alt und setzt an einer Stadtforschung an, die in großen Teilen von behavioristischen und humanistischen Ansätzen geprägt war. Seitdem haben sich nicht nur die Problemstellungen, Theorien und Methoden der Stadtforschung massiv verändert, auch die psychoanalytischen Geographien und nicht zuletzt die psychoanalytische Theorie werden hier nicht mehr hinreichend repräsentiert.

Insofern kommen Stadtforscher_innen bis auf Weiteres nicht umhin, sich den Originaltexten zuzuwenden, um einer Übersetzung der Psychoanalyse in die Stadtforschung nachzugehen. Dabei stellen insbesondere die Arbeiten von Lacan eine Herausforderung für den Einstieg in die Psychoanalyse dar. Nicht nur, dass Lacans fünfzigjähriges Schaffen eine Fülle theoretischer Überlegungen, Konzepte und Begrifflichkeiten bereithält, sein sperriger Stil und die Tatsache, dass sich seine Arbeiten auf über zwanzig (teils nicht veröffentlichte) Seminare und eine höchst anspruchsvolle Reihe an Schriften verteilen, lassen eine Erschließung seines Werkes zu einer Herausforderung werden. Vor diesem Hintergrund begründet sich Paul Kingsbury zufolge die zaghafte Annäherung an Lacan aus Sicht der anglophonen Geographie nicht so sehr in einer reflektierten Kritik, als in einer fehlenden Auseinandersetzung mit dessen Arbeiten (2003: 361). Eventuell lässt sich eine ähnliche These für das Verhältnis von Psychoanalyse und kritischer Stadtforschung im deutschsprachigen Raum aufstellen: Demnach sind es weniger die Inhalte der Psychoanalyse, die einen psychoanalytic turn hierzulande bisher ausbleiben lassen, als die fehlende Auseinandersetzung mit dessen Inhalten.

Bis heute hadert die Stadtforschung „zaghaft und misstrauisch“ (Pile 1996: 81) damit, ob und wie sie sich zur Psychoanalyse positionieren soll. Wenn wir jedoch, wie Ernesto Laclau behauptet, ,immer noch im Jahrhundert Freuds leben" und sich ein Großteil des zeitgenössischen Denkens bis heute daran abarbeitet, mit der „Entdeckung des Unbewussten zurechtzukommen“ (Laclau 2013: 93), dann kommt eine kritische Stadtforschung, die unserer Zeit gerecht werden will, nicht umhin, sich dieser Entdeckung ebenfalls anzunehmen.

Dieser Artikel wurde durch den Open-Access-Publikationsfonds der GoetheUniversität Frankfurt gefördert.

\section{Endnoten}

[1] Dass dies nicht notwendigerweise der Fall ist, hat Nikolai Roskamm kürzlich in seinem Buch Die unbesetzte Stadt (2017) herausgestellt. Hierin verhandelt Roskamm die Arbeiten von Freud und Lacan in einem Atemzug mit Autoren wie Marx/Engels, Harvey oder Lefebvre - ein Bezug, der in der deutschsprachigen Stadtforschung bislang ansonsten eher nicht gebräuchlich ist. Während Roskamm jedoch darauf abzielt, die Psychoanalyse in den Kanon einer postfundamentalistischen Stadttheorie zu integrieren, werde ich im Verlauf dieses Beitrags stärker dafür argumentieren, die Psychoanalyse als eigenständige Strömung innerhalb der kritischen Stadtforschung zu verhandeln. 
[2] Hierbei konzentriere ich mich hauptsächlich auf die beiden Strömungen, welche die psychoanalytische Geographie und Stadtforschung im anglophonen Raum am stärksten geprägt haben. Andere Bezüge, bspw. zu Alfred Lorenzer, Alexander Mitscherlich oder Georg Simmel, werden von mir deshalb außer Acht gelassen.

[3] Das bedeutet allerdings nicht, dass Lacan nicht auch von Marx beeinflusst war. Im Gegenteil findet sich bei Lacan eine ganz eigene Prägung des Freudomarxismus. Für eine systematische Analyse des Verhältnisses von Marx und Lacan, siehe Tomšič (2015).

[4] Mit Rückbezug auf die Debatten rund um die planetarische Urbanisierung lässt sich aus Sicht einer psychoanalytischen Stadtforschung folglich an ,der Stadt‘ als Konzept festhalten, insofern diese in den Bereich der Fantasie/Ideologie verlegt wird. Ein solcher Ansatz ist dem von Lefebvre in Teilen näher als der Ansatz von Brenner und Schmid. Schließlich spricht Lefebvre davon, dass ,die Stadt‘ in Zeiten einer massenhaften Urbanisierung zwar „keinem gesellschaftlichen Objekt mehr“ entspricht, jedoch als „Bild und - vor allem Ideologie“ bestehen bleibt (2014 [1970]: 65).

\section{Autor innen}

Lucas Pohl ist Humangeograph und beschäftigt sich mit der Vermittlung von Philosophie, Psychoanalyse und Geographie in Bezug auf Fragen der Stadt- und Raumtheorie, gebauten Umwelten und politischen Praxis.

pohl@geo.uni-frankfurt.de

\section{Literatur}

Adorno, Theodor W. (1962): Die revidierte Psychoanalyse. In: Max Horkheimer / Theodor W. Adorno (Hg.), Sociologica II. Reden und Vorträge. Frankfurt am Main: Suhrkamp, 119-138.

Althusser, Louis (2010 [1970]): Ideologie und ideologische Staatsapparate. Hamburg: VSA.

Benjamin, Walter (1991a): Gesammelte Schriften Band 5 Teil 1. Das Passagen-Werk. Frankfurt am Main: Suhrkamp.

Benjamin, Walter (1991b): Gesammelte Schriften Band 5 Teil 2. Das Passagen-Werk. Frankfurt am Main: Suhrkamp.

Berking, Helmuth / Löw, Martina (Hg.) (2008): Die Eigenlogik der Städte. Neue Wege für die Stadtforschung. Frankfurt am Main/New York: Campus.

Blum, Virginia / Nast, Heidi (2000): Jacques Lacan's two-dimensional subjectivity. In: Mike Crang / Nigel Thrift (Hg.), Thinking Space. London/New York: Routledge, 183-204.

Blum, Virginia / Secor, Anna J. (2011): Psychotopologies: Closing the circuit between psychic and material space. In: Environment and Planning D: Society and Space 29/6, 1030-1047.

Bondi, Liz (2009): Psychoanalytic theory. In: Derek Gregory / Ron Johnston / Geraldine Pratt / Michael J. Watts / Sarah Whatmore (Hg.), The Dictionary of Human Geography. Malden: Wiley-Blackwell, 595-597.

Brenner, Neil (2009): What is critical urban theory? In: City 13/2-3, 198-207.

Brenner, Neil / Schmid, Christian (2011): Planetary Urbanisation: In: Matthew Gandy (Hg.): Urban Constellations. Berlin: Jovis, 10-13.

Buck-Morss, Susan (2000): Dialektik des Sehens. Walter Benjamin und das Passagen-Werk. Frankfurt am Main: Suhrkamp.

Bullock, Nathan F. (2014): Lacan on urban development and national identity in a global city. Integrated resorts in Singapore. Identity of integrated resorts in Singapore. In: Singapore Journal of Tropical Geography 35/2, 213-227.

Bullock, Nathan F. (2018): A feminist psychoanalytic perspective on glass architecture in Singapore. In: Ilan Kapoor (Hg.), Psychoanalysis and the GlObal. Lincoln: University of Nebraska Press, 189-208.

Butler, Judith (1997): Körper von Gewicht. Die diskursiven Grenzen des Geschlechts. Frankfurt am Main: Suhrkamp.

Copjec, Joan (2002): Imagine There's No Woman. Ethics and Sublimation. Cambridge, Mass: MIT Press. 
Davidson, Mark (2010): Sustainability as ideological praxis: The acting out of planning's master-signifier. In: City 14/4, 390-405.

Dolar, Mladen (1998): Where Does Power Come From? In: New Formations 3, 79-92.

Featherstone, Mike (2005): Ruin Value. In: Journal for Cultural Research 9/3: 301-320.

Freud, Sigmund (1961 [1944]): Gesammelte Werke Band 15. Neue Folge der Vorlesungen zur Einführung in die Psychoanalyse. Frankfurt am Main: Fischer.

Freud, Sigmund (1966 [1947]): Gesammelte Werke Band 12. Werke aus den Jahren 1917-1920. Frankfurt am Main: Fischer.

Freud, Sigmund (1967 [1940]): Gesammelte Werke Band 13. Jenseits des Lustprinzips; Massenpsychologie und Ich-Analyse; Das Ich und das Es. Frankfurt am Main: Fischer.

Freud, Sigmund (1991) [1944]: Gesammelte Werke Band 14. Werke aus den Jahren 1925-1931. Frankfurt am Main: Fischer.

Gunder, Michael (2005): The production of desirous space: Mere fantasies of the utopian city? In: Planning Theory 4/2: 173-199.

Gunder, Michael (2008): Ideologies of certainty in a risky reality: Beyond the hauntology of planning”. In: Planning Theory 7/2, 186-206.

Gunder, Michael (2011): A metapsychological exploration of the role of popular media in engineering public belief on planning issues. In: Planning Theory 10/4, 325-343.

Gunder, Michael / Hillier, Jean (2009): Planning in Ten Words or Less: A Lacanian entanglement with spatial planning. Farnham: Ashgate.

Hillier, Jean (2003): ,Agon'izing over consensus: Why Habermasian ideals cannot be ,real'. In: Planning Theory 2/1, 37-59.

Hillier, Jean / Gunder, Michael (2003): Planning fantasies? An exploration of a potential Lacanian framework for understanding development assessment planning. In: Planning Theory 2/3, 225-248.

Hillier, Jean / Gunder, Michael (2005): Not over your dead bodies! A Lacanian interpretation of urban planning discourse and practice. In: Environment and Planning A: Economy and Space 37, 1049-1066.

Kapoor, Ilan (Hg.) (2018): Psychoanalysis and the GlObal. Lincoln: University of Nebraska Press.

Kingsbury, Paul (2003): Psychoanalysis, a gay spatial science? In: Social \& Cultural Geography 4/3, 347-367.

Kingsbury, Paul (2004): Psychoanalytic approaches. In: James S. Duncan / Nuala C. Johnston / Richard H. Schein (Hg.), A Companion to Cultural Geography. New Jersey: Blackwell, 108-120.

Kingsbury, Paul (2007): The extimacy of space. In: Social \& Cultural Geography 8/2, 235-258.

Kingsbury, Paul (2009): Psychoanalytic Theory/Psychoanalytic Geographies. In: Rob Kitchin / Nigel Thrift (Hg.), International Encyclopedia of Human Geography. Oxford: Elsevier, 487-494.

Kingsbury, Paul (2017): Uneasiness in culture, or negotiating the sublime distances towards the big Other. In: Geography Compass 11/6, e12316.

Kingsbury, Paul / Pile, Steve (Hg.) (2014a): Psychoanalytic Geographies. Farnham: Ashgate.

Kingsbury, Paul / Pile, Steve (2014b): When in Rome... In: Paul Kingsbury / Steve Pile (Hg.), Psychoanalytic Geographies. Farnham: Ashgate, xvii-xxii.

Lacan, Jacques (2006): Der Triumph der Religion, welchem vorausgeht: Der Diskurs an die Katholiken. Wien/Berlin: Turia + Kant.

Lacan, Jacques (2010 [2004]): Die Angst. Das Seminar. Buch X. Wien/Berlin: Turia + Kant. Lacan, Jacques (2011 [1955]): Das Freud'sche Ding. Wien/Berlin: Turia + Kant.

Lacan, Jacques (2015a [1979]): Struktur. Andersheit. Subjektkonstitution. Köln: August.

Lacan, Jacques (2015b [1975]): Freuds Technische Schriften. Das Seminar. Buch I. Wien/ Berlin: Turia + Kant.

Lacan, Jacques (2015c [1975]): Encore. Das Seminar. Buch XX. Wien/Berlin: Turia + Kant.

Lacan, Jacques (2015d [1973]): Die vier Grundbegriffe der Psychoanalyse. Das Seminar. Buch XI. Wien/Berlin: Turia + Kant.

Lacan, Jacques (2016 [1986]): Die Ethik der Psychoanalyse. Das Seminar. Buch VII. Wien/ Berlin: Turia + Kant. 
Laclau, Ernesto (2013): Identität und Hegemonie. Die Rolle der Universalität in der Konstitution von politischen Logiken. In: Judith Butler / Ernesto Laclau / Slavoj Žižek (Hg.), Kontingenz Hegemonie Universalität. Aktuelle Dialoge zur Linken. Wien/Berlin: Turia + Kant, 57-111.

Lefebvre, Henri (2000 [1974]): La production de l'espace. Paris: Anthropos.

Lefebvre, Henri (2014 [1970]): Die Revolution der Städte. Hamburg: CEP Europäische Verlagsanstalt.

Löw, Martina (2008): Soziologie der Städte. Frankfurt am Main: Suhrkamp.

Löw, Martina (2010): Stadt- und Raumsoziologie. In: Georg Kneer / Markus Schroer (Hg.): Handbuch Spezielle Soziologien. Wiesbaden: VS, 605-622.

Martin, Lauren / Secor, Anna J. (2014): Towards a post-mathematical topology. In: Progress in Human Geography 38/3, 420-438.

Nichols, Joshua (2008): Lacan, the city, and the utopian symptom. In: Space and Culture $11 / 4,459-474$.

Parker, Simon (2004): Urban theory and the Urban Experience: Encountering the city. London/New York: Routledge.

Philo, Chris / Parr, Hester (2003): Introducing psychoanalytic geographies. In: Social \& Cultural Geography 4/3, 283-293.

Pile, Steve (1991): Practising interpretative geography. In: Transactions of the Institute of British Geographers 16/4, 458-469.

Pile, Steve (1993): Human agency and human geography revisited: A critique of, new models of the self. In: Transactions of the Institute of British Geographers 18/1, 122-139.

Pile, Steve (1996): The Body And The City: Psychoanalysis, Space, and Subjectivity. London/ New York: Routledge.

Pile, Steve (2000): Sleepwalking in the modern city: Walter Benjamin and Sigmund Freud in the world of dreams. In: Gary Bridge / Sophie Watson (Hg.), A Companion To The City. Malden: Blackwell, 75-86.

Pile, Steve (2005a): Real Cities: Modernity, Space, and the Phantasmagorias of City Life. London: Sage.

Pile, Steve (2005b): Spectral Cities: Where the Repressed Returns and Other Short Stories. In: Jean Hillier / Emma Rooksby (Hg.), Habitus: A sense of place. Sydney: Ashgate, 219-239.

Pile, Steve (2011): Intensities of feeling: Cloverfield, the uncanny, and the always near collapse of the city. In: Gary Bridge / Sophie Watson (Hg.), The New Blackwell Companion to the City. Chichester: Blackwell, 288-303.

Pohl, Lucas (2017): Imaginary politics of the branded city: Right-wing terrorism as a mediated object of stigmatization. In: Paul Kirkness / Andreas Tijé-Dra (Hg.), Negative Neighbourhood Reputation and Place Attachment: The Production and Contestation of Territorial Stigma. London/New York: Routledge, 27-41.

Pohl, Lucas (2018a): Architectural enjoyment. Lefebvre and Lacan. In: Ilan Kapoor (Hg.), Psychoanalysis and the GlObal. Lincoln: University of Nebraska Press, 142-163.

Pohl, Lucas (2018b): Die unsterbliche Stadt: Das Unbehagen in den Wolkenkratzern von Detroit. In: dérive 70, 13-18.

Pohl, Lucas (2018c): Angsträume. Lacan und die Leere des Schauplatzes. In: RISS Materialien, Ent-täuschung des Subjekts. Angst in Philosophie, Psychoanalyse und Kultur. Sonderausgabe des RISS Zeitschrift für Psychoanalyse 4, 65-83.

Pohl, Lucas (2019a): Psychoanalysis. In: Audrey Kobayashi (Hg.), International Encyclopedia of Human Geography. Second Edition. Amsterdam: Elsevier, im Erscheinen.

Pohl, Lucas (2019b): Object-disoriented geographies: The Ghost Tower of Bangkok and the topology of anxiety. In: cultural geographies, online first, 1-14.

Pohl, Lucas (2019c): The Sublime Object of Detroit. In: Social \& Cultural Geography, im Erscheinen.

Proudfoot, Jesse (2009) Geography and Psychoanalysis. In: Barney Warf (Hg.), Encyclopedia of Geography. Los Angeles: Sage, 2299-2301.

Proudfoot, Jesse (2011) The Anxious Enjoyment of Poverty: Drug Addiction, Panhandling, and the Spaces of Psychoanalysis. Burnaby: Simon Fraser University.

Proudfoot, Jesse (2017): The libidinal economy of revanchism: Illicit drugs, harm reduction, and the problem of enjoyment. In: Progress in Human Geography 43/2, 214-234. 
Roskamm, Nikolai (2017): Die unbesetzte Stadt. Postfundamentalistisches Denken und das urbanistische Feld. Basel: Birkhäuser.

Secor, Anna J. (2013): Topological City. Urban Geography 34/4, 430-444.

Social \& Cultural Geography (2003): Special Issue Psychoanalytic Geographies, 4: 283-399.

Stavrakakis, Yannis (2009): Psychoanalysis and ideology: Comment on R.D. Hinshelwood. In: Psychoanalysis, Culture \& Society 14/2, 149-163.

The Professional Geographer (2010): Special Issue Psychoanalytic Methodologies in Geography, 62/4, 478-533.

Tomšič, Samo (2015): The Capitalist Unconscious. Marx and Lacan. London/New York: Verso.

Westin, Sara (2014): The Paradoxes of Planning: A Psycho-Analytical Perspective. Farnham: Ashgate.

Wilson, Japhy / Bayón, Manuel (2017): Fantastical materializations: Interoceanic infrastructures in the Ecuadorian Amazon. Environment and Planning D: Society and Space 35/5, 836-854.

Žižek, Slavoj (1989): The Sublime Object of Ideology. London/New York: Verso.

Žižek, Slavoj (1991): Looking Awry: An Introduction to Jacques Lacan Through Popular Culture. Cambridge: MIT Press.

Zupančič, Alenka (2009): Warum Psychoanalyse? Drei Interventionen. Zürich/Berlin: diaphanes.

\section{The Urban Unconscious. Psychoanalysis and critical urban studies}

At the beginning of the 1990s, anglophone geographies started to investigate the relationship between psychoanalysis and the city. In the beginning of the 20oos, geographers announced a "psychoanalytic turn". Sub-disciplines such as "psychoanalytic geographies" and "psychoanalytic planning theory" were founded and have started to become established components within the scholarly debates on cities in the anglophone world. There has been no such "turn" in the German-speaking hemisphere. Therefore, this paper retraces the potential of psychoanalytic urban studies. The author follows the idea of an urban unconscious. The urban unconscious characterizes a constitutive disruption that is inscribed into the topology of the city and ultimately makes it impossible to speak of the city as a coherent object (of urban studies). Starting from this impossibility, the paper examines the fantasies surrounding the social, political and material environments of the city. From a psychoanalytic standpoint, fantasies play a central role in providing the city with an illusion of consistency and maintaining a distance towards the urban unconscious. They allow us to imagine the city, to feel, and speak about it. The paper concludes with a few words about the general challenges for critical urban scholars to engage with psychoanalysis. 